Rev. Biol. Trop., 47(1-2): 33-43, 1999

www.ucr.ac.cr www.ots.ac.cr www.ots.duke.edu

\title{
Reabsorción de nitrógeno y fósforo foliar en árboles de bosques montanos en los Andes centrales de Colombia
}

\author{
Miguel Vera ${ }^{1}$, Jaime Cavelier ${ }^{2}$ y Johanna Santamaría ${ }^{3}$ \\ Departamento de Biología, Pontificia Universidad Javeriana, \\ Departamento de Ciencias Biológicas, Universidad de los Andes, Bogotá. \\ Correspondencia: J. Cavelier, Laboratorio de Ecología Vegetal, Departamento de Ciencias Biológicas, Universidad de \\ Los Andes, Carrera 1 No. 18A-70, Bogotá, Colombia. Fax 2841890. Internet: jcavelie@ uniandes.edu.co
}

Recibido 27-VI-1998. Corregido 22-X-1998. Aceptado 10-XI-1998.

\begin{abstract}
Mature leaves and litterfall were collected between November 1992 and February 1993 from one to three individuals of five canopy tree species of a lower montane rain forest (LMRF) at $1850 \mathrm{~m}$. and five canopy tree species of an upper montane rain forest (UMRF) at $2800 \mathrm{~m}$. in the Central Andes of Colombia, to determine the concentrations and percentage retranslocation of nitrogen and phosphorus. The availability of these two nutrients was measured in the soils, by means of extracts of $\mathrm{NH}_{3}-\mathrm{NH}_{4}, \mathrm{NO}_{2}-\mathrm{NO}_{3}$ and $\mathrm{PO}_{4}$, and incubations of surface soil samples $(0-10 \mathrm{~cm})$ to measure the rates of nitrogen mineralization and nitrification. In the species of the LMRF the average concentration of $\mathrm{N}$ in mature leaves ( $1.19 \%$ dry weight) and litterfall $(0.87 \%)$ was significantly higher than in mature leaves $(0.90 \%)$ and litterfall $(0.59 \%)$ of the tree species of the UMRF. Percentage retranslocation of $\mathrm{N}$ and $\mathrm{P}$ were not significantly different between the species of these two forests. In spite of this, P retranslocation tended to be higher in the species of the LMRF where the available P in the soil was significantly lower (3.2 ppm vs. $9.0 \mathrm{ppm}$ ). A negative and statistically significant correlation was found between the percentage retranslocation of $\mathrm{P}$ and the concentration of this element in mature leaves of 12 different tropical montane forests. These results suggest that percentage retranslocation of foliar $\mathrm{P}$ in montane forests, could be partially controlled by the availability of this element. There were no statistically significant correlations between percentage retranslocation of $\mathrm{N}$ and $\mathrm{P}$ and specific leaf area for the ten tree species of this study. The lack of correlation may be the result of a small sample size and should be explored with a higher number of species.
\end{abstract}

Key words: Retranslocation, reabsortion, tropical montane forests, nitrogen mineralization, phosphorus, cloud forest, altitudinal gradient.

En gradientes altitudinales húmedos de montañas tropicales, se reconocen tres tipos de formaciones boscosas: bosques húmedos de tierras bajas, bosques montanos bajos y bosques montanos altos (Grubb 1977). A lo largo de este gradiente se observan cambios en la estructura y funcionamiento de los bosques, por ejemplo la reduccción del tamaño medio de los árboles, de las hojas y de la productividad (Leigh 1975, Grubb 1977, Cave- lier 1996). Estas diferencias en la vegetación están correlacionadas con cambios climáticos y edáficos, como la disminución de la temperatura media del aire y del suelo (Schubert \& Medina 1982, van der Hammen 1984) y la acumulación de materia orgánica (Alexander \& Pichott 1979, Vitousek et al. 1988, Grieve et al. 1990). La baja fertilidad de los suelos en bosques montanos tropicales (Marrs et al. 1988, Vitousek et al.1988) se ve reflejada en 
una baja concentración (\% por unidad de peso) de nitrógeno y fósforo foliar (Tanner 1977a), una baja productividad (Grubb 1977) y una alta eficiencia de uso de nutrientes sensu Vitousek (1984). En este ambiente, mecanismos de conservación de nutrientes como la reabsorción de elementos foliares móviles (i.e. nitrógeno y fósforo) antes de la caída de las hojas (Chapin 1980, Charley \& Richards 1983) tendrían valor adaptativo. A pesar de la importancia potencial de este mecanismo de conservación de nutrientes existe muy poca información específica en árboles de bosques montanos tropicales (Edwards 1982, Grubb \& Edwards 1982).

Las especies de árboles de los bosques montanos pueden presentar hojas escleromórficas ("pachyphylls" sensu Grubb 1986) o mesófilas (Tanner \& Kapos 1982, Sugden 1985). Las hojas escleromórficas son por lo general gruesas, con valores bajos de área foliar específica ( $\mathrm{cm} 2 / \mathrm{g}$ ), de textura coriácea y baja concentración de nutrientes por unidad de pesos debido a la acumulación de compuestos de carbono (Medina 1984). En contraste, las hojas "mesófilas" son relativamente delgadas y con concentraciones mayores de nutrientes foliares por unidad de peso seco. El escleromorfismo está asociado a otras características foliares como la longevidad y las bajas tasas de crecimiento (Chapin 1980, Grubb 1986) rasgos que parecen haber sido seleccionados en ambientes oliogotróficos (Loveless 1961, 1962, Medina 1984). Si el escleromorfismo está asociado a condiciones de baja fertilidad, la reabsorción de nutrientes en estas especies debería ser más eficiente que en especies mesófilas. Esta relación no se ha establecido con especies de bosques montanos tropicales, como se si logró con especies de turbera en la zona templada (Small 1972). En sabanas y bosques de tierras bajas tropicales, comparaciones entre los porcentajes de reabsorción de nitrógeno y fósforo foliar entre especies caducifolias y siempreverdes (más escleromórficas), no muestran una relación entre escleromorfismo y las tasas de reabsorción (Medina 1984).

En este estudio, se midieron las tasas de reabsorción de nitrógeno y fósforo foliar en un total de diez especies de árboles de bosques montanos altos y bajos. Si a mayor altitud disminuye la fertilidad de los suelos y la reabsorción es un mecanismo importante para la conservación de nutrientes, las especies del bosque montano alto, deberían presentar un mayor porcentaje de reabsorción de nitrógeno y fósforo. Además, si las hojas escleromórficas tienen bajas concentraciones de nitrógeno y fósforo y estas han sido seleccionadas en suelos oligotróficos, sería de esperar que estas especies reabsorban un mayor porcentaje de nutrientes antes de la caída de las hojas.

\section{MATERIALES Y MÉTODOS}

Área de estudio y especies: este estudio fue realizado en el Parque Regional Natural Ucumarí (Risaralda, Colombia), en la vertiente occidental de la Cordillera Central de los Andes colombianos $\left(4^{\circ} 40^{\prime} \mathrm{N} ; 75^{\circ} 31^{\prime} 0\right)$. El trabajo de campo se realizó en un Bosque Montano Bajo (BMB) a 1850 m. s. n. m. alrededor de la Estación La Suiza y en un Bosque Montano Alto (BMA) a 2800 m. s. n. m. alrededor de la Estación La Pastora. El BMB (sensu Grubb 1977) tiene una altura media de $20 \mathrm{~m}$ y el BMA de $17 \mathrm{~m}$. (Cleef et al. 1984). Los suelos de esta parte de la Cordillera Central colombiana, se han desarrollado a partir de cenizas volcánicas y otros piroclastos producto de la actividad volcánica durante el Holoceno (Thouret 1983). Los BMB crecen sobre suelos ferrisoles ándicos humíferos, desaturados y lixiviados con horizonte ocre u óxico mientras que los BMA crecen sobre suelos párdos ándicos más o menos ácidos (Thouret 1989). La precipitación media anual a lo largo de este gradiente altitudinal varía entre 3000 y 1988 mm, con un patrón bimodal y con épocas de menores precipitaciones durante los meses de enero-febrero y julio-agosto. En cada tipo de bosque se recolectaron, entre noviembre de 1992 y febrero de 1993, hojas maduras y hojarasca en uno a tres individuos de cinco especies de árboles de dosel (Cuadro 1). La recolección de hojas en el dosel se llevó a 
VERA et. al.: Reabsorción de nitrógeno y fósforo

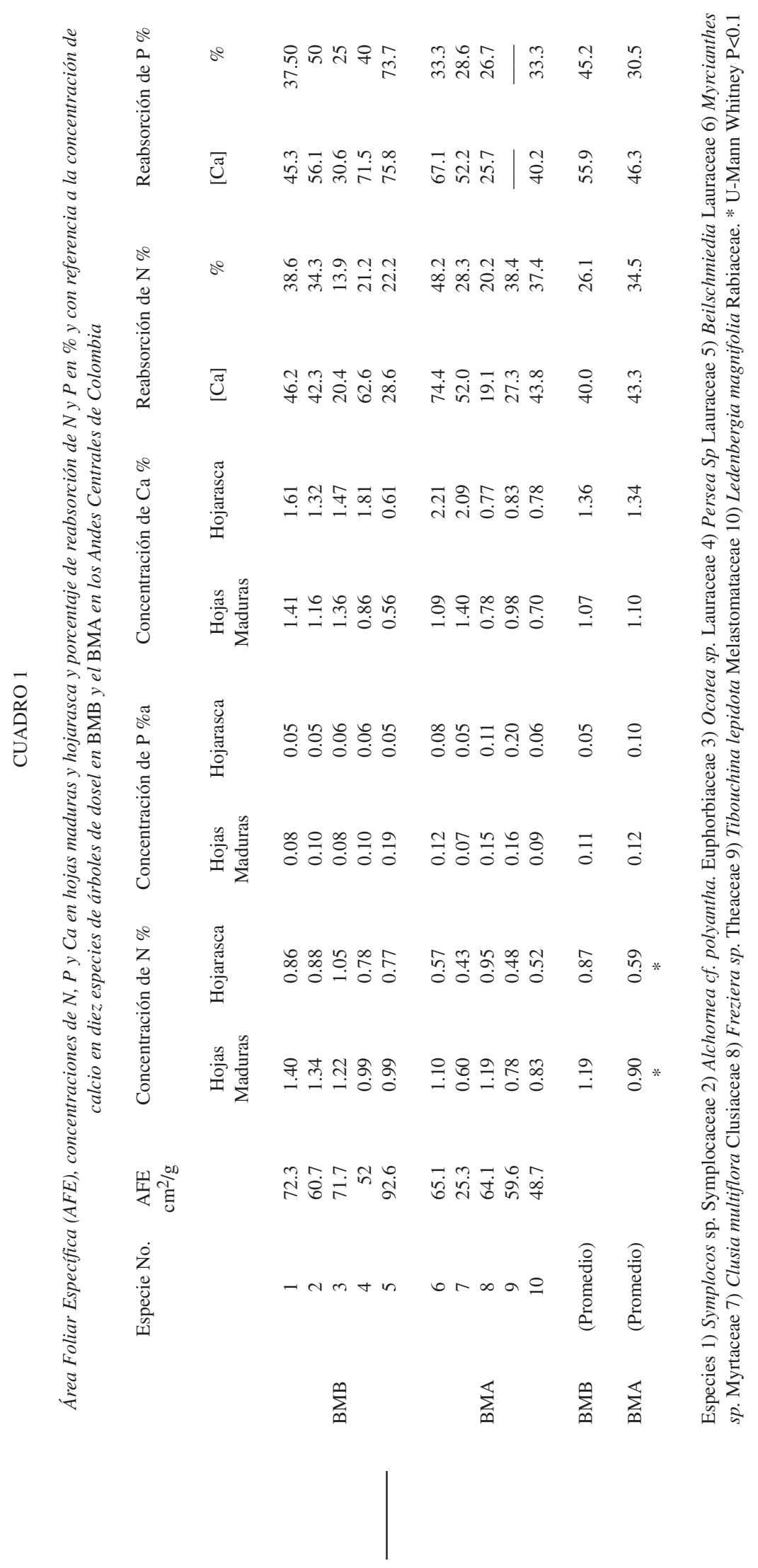


cabo mediante técnicas de escalada de árboles. La recolección de hojarasca se realizó mediante 40 trampas de 0.5 x $0.5 \mathrm{~m}$. Estas se construyeron con marcos de tubería de PVC de $1.0 \mathrm{~cm}$ de diámetro, con malla de fibra de vidrio y se colocaron a un metro sobre el nivel del suelo. Las trampas se ubicaron debajo de aquellos individuos seleccionados para la recolección de hojas maduras y se revisaron a diario para garantizar que las hojas recolectadas estuvieran recién caídas. Las muestras de hojas recolectadas en el dosel y la hojarasca se secaron a $60{ }^{\circ} \mathrm{C}$ por $48 \mathrm{~h}$ antes de realizarse los análisis químicos. Para hojas maduras y hojarasca se determinó el área foliar específica $\left(\mathrm{cm}^{2} / \mathrm{g}\right)$ que se obtiene de la relación superficie foliar fresca/peso seco (Stoker 1931).

Análisis foliares y tasas de reabsorción: se determinaron las concentraciones de nitrógeno en hojas maduras y hojarasca de las especies seleccionadas usando el método Kjeldahl. Para la determinación de las concentraciones de $\mathrm{P}$ y $\mathrm{Ca}$ se utilizaron los métodos descritos en Allen et al. 1974.

Para calcular las tasas de reabsorción se utilizó la fórmula:

$\%($ Reab. $)=([$ Nut] Hojas maduras-[Nut] Hojarasca $]) /$ ([Nut] Hojas maduras])

donde ([N] Hojas maduras) es la concentración en porcentaje (\% de peso seco) del nutriente en hojas maduras y ([Nut] Hojarasca) es la concentración en porcentaje del nutriente en hojarasca (Del Arco et al. 1991).

Considerando que cantidades variables de materia orgánica y nutrientes minerales son retiradas antes de la caída de las hojas, el porcentaje de reabsorción también se calculó teniendo en cuenta la concentración de calcio como referencia, un elemento inmóvil una vez incorporado en los tejidos. Por lo tanto, el porcentaje de N y P reabsorbidos se estimó con la siguiente fórmula: (modificado de Vitousek \& Sanford 1986)

(\%) Reab. $=(1-([\mathrm{Nut}]$ en Hojarasca / [Ca] en hojarasca $)$ / ([Nut.] en H. maduras / [Ca] en H. maduras) *100.
Medición de la disponibilidad de $\mathbf{N}$ y $\mathbf{P}$ en el suelo: para determinar las concentraciones de $\mathrm{NH}_{3}-\mathrm{NH}_{4}, \mathrm{NO}_{2}-\mathrm{NO}_{2}$ y $\mathrm{PO}_{4}$, así como las tasas de mineralización de nitrógeno y nitrificación se tomaron quince muestras de suelo $(0-10 \mathrm{~cm})$ en cada tipo de bosque mediante un taladro cilíndrico de $4.6 \mathrm{~cm}$ de diámetro y $10 \mathrm{~cm}$ de largo. El amonio y los nitratos se extrajeron tomando $10 \mathrm{~g}$ de cada muestra y usando $100 \mathrm{ml} 2 \mathrm{M}$ de KCL como solución de extracción. Las submuestras se lavaron en la solución por un minuto, se dejaron en reposo durante $6 \mathrm{~h}$ y se filtraron en papel Whatman No. 44 (Robertson 1984).

Para extraer el fósforo se usó fluoruro áci-

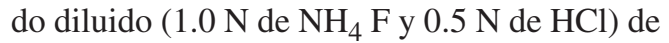
acuerdo a Olsen y Sommers (1982). Se utilizaron $2 \mathrm{~g}$ de suelo y se lavaron con $2 \mathrm{ml}$ de solución durante 5 minutos. Luego se filtró.

Las tasas de mineralización del nitrógeno y la nitrificación neta fueron medidas en cada bosque utilizando incubaciones in situ de quince muestras de suelo de aproximadamente 100 g cada una durante 30 días. Antes y después de la incubación, el amonio y el nitrato fueron extraídos de 10 g de suelo de cada una de las quince muestras. La tasa de mineralización de nitrógeno se determinó aplicando la fórmula: (Concentración final de $\left[\mathrm{NH}_{4}-\mathrm{N}+\mathrm{NO}_{3}-\mathrm{N}+\right.$ $\left.\mathrm{NO}_{2}-\mathrm{N}\right]-$ Concentración inicial de $[\mathrm{NH} 4-\mathrm{N}+$ $\left.\mathrm{NO}_{3}-\mathrm{N}+\mathrm{NO}_{2}-\mathrm{N}\right]$ ) y la tasa de nitrificación neta aplicando la fórmula (Concentración final de $\left[\mathrm{NO}_{3}-\mathrm{N}+\mathrm{NO}_{2}-\mathrm{N}\right]-$ Concentración inicial de $\left.\left[\mathrm{NO}_{3}-\mathrm{N}+\mathrm{NO}_{2}-\mathrm{N}\right]\right)$ de acuerdo con Robertson y Vitousek (1981) y Robertson (1984). El amonio y el nitrato de las muestras de suelo se preservaron en campo con $1 \mathrm{mg} / \mathrm{l}$ de cloruro de mercurio (Robertson 1984) y su concentración fue determinada por medio de espectofotometría. Para la determinación de $\mathrm{NO}_{3}-\mathrm{N}+\mathrm{NO}_{2}-\mathrm{N}$, el nitrato fue reducido a nitrito, y el nitrito total fue determinado por espectrofotometría.

Análisis estadístico de datos: la concentración de nutrientes en hojas, los porcentajes de reabsorción de nitrógeno y fósforo, así como las concentraciones de nitrógeno en el suelo y las tasas de mineralización y nitrificación, 
fueron comparadas por medio de la prueba de U-Mann Whitney. Además, se hicieron correlaciones mediante la prueba de Spearman entre el porcentaje de reabsorción de nutrientes, el área foliar específica y la concentración de nitrógeno y fósforo en hojas maduras. Dado el bajo número de especies se usó un nivel de significancia de $\mathrm{p}<0.10$.

\section{RESULTADOS}

Concentraciones de $\mathbf{N}$ y $\mathbf{P}$ en hojas maduras y hojarasca: en las especies del BMB la concentración promedio de $\mathrm{N}$ (\% de peso seco) en hojas maduras $(1.19 \%)$ y hojarasca $(0.87 \%)$ fue mayor (U-Mann Whitney $\mathrm{p}<0.10)$ que en hojas maduras $(0.90 \%)$ y hojarasca $(0.59 \%)$ de las especies del BMA. No hubo diferencias estadísticamente significativas (U-Mann Whitney p>0.10) en las concentraciones de $\mathrm{P}$ y $\mathrm{Ca}$ de hojas y hojarasca entre estos dos bosques (Cuadro 1).

Tasas de reabsorción: el porcentaje de reabsorción de $\mathrm{N}$ no fue significativamente diferente (U-Mann Whitney, $\mathrm{p}>0.10$ ) entre las especies de BMA $(34.5+/-5.3 ; n=5)$ y las especies de BMB $(26.0+/-5.1 ; n=5)$. Cuando la reabsorción de $\mathrm{N}$ fue expresada usando la concentración de $\mathrm{Ca}$ como referencia, las diferencias entre estos dos bosques fueron aún menores (Cuadro 1). El porcentaje de reabsorción de $\mathrm{P}$, no fue significativamente diferente (U-Mann Whitney, $\mathrm{p}>0.10$ ) entre las especies del BMB (45.2+/- 9.1; $\mathrm{n}=4)$ y las especies del BMA (30.5 +/- 19.0; $\mathrm{n}=4)$. Cuando la reabsorción de $\mathrm{P}$ fue calculada usando la concentración de Ca como referencia, esta diferencia se hizo mayor pero no fue significativa. Para Tibouchina lepidota, el porcentaje de reabsorción de $\mathrm{P}$ no pudo ser calculado, pues las concentraciones en hojarasca fueron mayores que en hojas maduras.

Cuando se consideraron las diez especies en conjunto, no se presentaron correlaciones estadísticamente significativas entre el porcentaje de reabsorción de N o P y área foliar específica o la concentración de estos elementos en hojas maduras.

Disponibilidad de $\mathbf{N}$ y $\mathbf{P}$ en el suelo: las concentraciones de $\mathrm{NO}_{3}$ en el suelo superficial $(0-10 \mathrm{~cm})$ fueron significativamente mayores (U-Mann-Whitney; $\mathrm{p}<0.1$ ) en el BMA que en el BMB (Cuadro 2). Las concentraciones de amonio, así como las tasas de mineralización del nitrógeno y nitrificación, no fueron significativamente diferentes entre los suelos de los dos tipos de bosques. La concentración de P disponible (Bray II) en el suelo fue significativamente mayor (U-MannWhitney; $\mathrm{p}<0.10$ ) en el BMA que en el BMB (Cuadro 2).

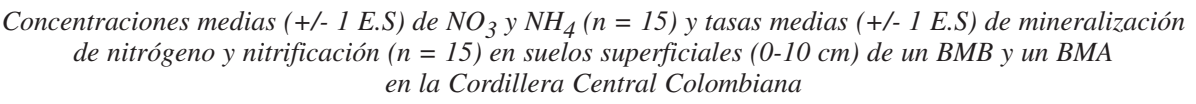
en la Cordillera Central Colombiana

Concentraciones

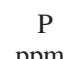

ppm

$\mathrm{BMB}$

$3.2+/-0.7$

$9.0+/-1.9 *$

Tasas

BMA

$9.0+/-1.9 * \quad 83.0+/-29 *$

$$
\mathrm{NO}_{3}
$$

$\mu \mathrm{g} / \mathrm{g}$

$\mathrm{NH}_{4}$

$22.9+/-3.9$

$28.6+/-7.3$

* U-Mann Whitney $\mathrm{P}<0.1$. Nit= nitrificación; Min= mineralización. 


\section{DISCUSION}

En este trabajo se presentan las tasas de reabsorción de $\mathrm{N}$ y $\mathrm{P}$ foliar en diez especies de árboles de bosques montanos tropicales. A pesar de que solo se tienen de una a tres réplicas por especies, este es uno de los pocos estudios donde se tiene información sobre especies individuales (Grubb \& Edwards 1982, Edwards 1982), ya que en la mayoría de los trabajos en bosques tropicales, la información sobre reabsorción ha sido derivada de comparaciones entre hojas y hojarasca a nivel del bosque (Tanner 1977a,b, Steinhardt 1979, Fassbender \& Grimm 1981, Medina et al. 1981, Weaver 1986, Proctor et al. 1989, Veneklass 1991). A pesar de que la reabsorción de nutrientes foliares antes de la caída de las hojas se ha mencionado como un mecanismo de conservación de nutrientes en bosques tropicales (Jordan 1985), son pocos los estudios que de una u otra forma proporcionan información cuantitativa sobre esta posible ruta de circulación de nutrientes (i.e. Vitousek \& Sanford 1986).

Concentraciones de N y P en hojas maduras y hojarasca: la concentración promedio de nitrógeno en hojas maduras 04ะ008) y hojarasca $0.73 \pm 0.07$ de las diez especies estudiadas, está en el límite inferior de los valores reportados para otros bosques montanos tropicales (Cuadro 3). Solo los valores medidos para siete especies de árboles en el bosque enano nublado de Pico del Este, Puerto Rico, son similares (Cuadro 3). En contraste, la concentración de fósforo en hojas $0.11 \pm 0.01$ y hojarasca $0.08 \pm 0.01$ en la Cordillera Central colombiana, esta en el límite superior (Cuadro 3). A pesar de estos valores extremos, el porcentaje promedio de reabsorción de $\mathrm{N} 30.3 \pm 3.40$ y $\mathrm{P}$ $38.6 \pm 5.10$ de las diez especies del presente estudio está dentro del rango de valores de bosques montanos tropicales $(\mathrm{N}=16-42, \mathrm{P}=25-69$, Cuadro3) y de tierras bajas $(\mathrm{N}=11-35, \mathrm{n}=5$ bosques, $\mathrm{P}=29-50, \mathrm{n}=5$ bosques, Cavelier 1996). Los únicos valores más altos de reabsorción de $\mathrm{N}$ y $\mathrm{P}$ foliar son los de cuatro especies de árboles de sabana $(\mathrm{N}=44.5 \% ; \mathrm{P}=57.5$
CUADRO 3

\begin{tabular}{|c|c|c|c|c|}
\hline \multicolumn{5}{|c|}{$\begin{array}{c}\text { Concentración (\% de peso seco) de } \\
\text { en hojas maduras de dosel y hojarc } \\
\text { de montaña }\end{array}$} \\
\hline & \multicolumn{2}{|c|}{$\begin{array}{c}\text { Concentración } \\
\%\end{array}$} & \multicolumn{2}{|c|}{$\begin{array}{c}\text { Reabsorción } \\
\%\end{array}$} \\
\hline & $\mathrm{N}$ & $\mathrm{P}$ & $\mathrm{N}$ & $\mathrm{P}$ \\
\hline \multicolumn{5}{|l|}{ Colombia (1) } \\
\hline \multicolumn{5}{|l|}{ BMA } \\
\hline Hojas maduras & 1.47 & 0.09 & & \\
\hline Hojarasca & 0.90 & 0.03 & 39 & 67 \\
\hline \multicolumn{5}{|l|}{ BMB } \\
\hline Hojas maduras & 1.78 & 0.127 & & \\
\hline Hojarasca & 1.08 & 0.070 & 39 & 45 \\
\hline \multicolumn{5}{|l|}{ Jamaica (2) } \\
\hline Hojas Maduras & 1.30 & 0.068 & & \\
\hline Hojarasca & 0.76 & 0.030 & 42 & 56 \\
\hline \multicolumn{5}{|c|}{ Nueva Guinea (3) } \\
\hline Hojas maduras & 1.32 & 0.086 & & \\
\hline Hojarasca & 1.11 & 0.059 & 16 & 31 \\
\hline \multicolumn{5}{|l|}{ Puerto Rico (4) } \\
\hline Hojas maduras & 0.99 & 0.063 & & \\
\hline Hojarasca & 0.77 & 0.024 & 22 & 62 \\
\hline \multicolumn{5}{|l|}{ Sabah (5) } \\
\hline \multicolumn{5}{|l|}{ a $610 \mathrm{~m}$} \\
\hline Hojas maduras & 1.45 & 0.047 & & \\
\hline $\begin{array}{l}\text { Hojarasca } \\
\text { a } 790\end{array}$ & 1.05 & 0.017 & 28 & 64 \\
\hline Hojas maduras & 1.72 & 0.054 & & \\
\hline $\begin{array}{l}\text { Hojarasca } \\
\text { a } 870\end{array}$ & 1.05 & 0.017 & 39 & 69 \\
\hline Hojas maduras & 1.34 & 0.046 & & \\
\hline Hojarasca & 0.83 & 0.020 & 38 & 57 \\
\hline \multicolumn{5}{|l|}{ Venezuela (6) } \\
\hline Hojas maduras & 1.74 & 0.08 & & \\
\hline Hojarasca & 1.20 & 0.06 & 31 & 25 \\
\hline \multicolumn{5}{|l|}{ Venezuela (7) } \\
\hline Hojas maduras & 1.64 & 0.111 & & \\
\hline Hojarasca & 1.15 & 0.062 & 30 & 44 \\
\hline \multicolumn{5}{|c|}{ Colombia (Este Estudio) } \\
\hline Hojas maduras & 1.18 & 0.11 & & \\
\hline Hojarasca & 0.87 & 0.05 & 26 & 45 \\
\hline \multicolumn{5}{|l|}{ BMA } \\
\hline Hojas maduras & 0.90 & 0.12 & & \\
\hline Hojarasca & 0.59 & 0.10 & 34 & 30 \\
\hline
\end{tabular}

(1) Veneklaas (1991); (2) Tanner, (1977 a,b); (3) Grubb \& Edwards (1982); Edwards (1982); (4) Medina Cuevas \& Weaber (1981); Weaver, et al. (1986); (5) Proctor et al. (1989); (6) Fassbender \& Grimm (1981); (7) Steinhardt (1979). 
\%, Montes \& Medina 1977), un ecosistema con suelos muy pobres (Sarmiento 1984).

Reabsorción de $\mathbf{N}$ y $\mathbf{P}$ foliar: no se presentaron diferencias estadísticamente significativas en los porcentajes de reabsorción de $\mathrm{N}$ foliar entre las especies de los BMB y BMA, ni diferencias en las tasas de mineralización y nitrificación a estas dos altitudes. Los valores de disponibilidad de nitrógeno en el suelo presentados en este estudio, están dentro del rango de valores observados en otros bosques tropicales (Marrs et al. 1988, Vitousek et al. 1988, Robertson 1989). De igual forma, no se presentaron correlaciones estadísticamente significativas entre los porcentajes de reabsorción de $\mathrm{N}$ foliar y las concentraciones de $\mathrm{N}$ en hojas maduras tanto para las diez especies de este estudio como para los bosques montanos en su conjunto (Cuadro 3). Al parecer, los porcentajes de reabsorción de $\mathrm{N}$ foliar son muy similares entre diferentes tipos de bosques montanos y no varían consistentemente con la disponibilidad de nutrientes, estimada con las medidas de concentración en hojas y suelos (Cavelier 1996). Estudios en ecosistemas templados muestran resultados variables con respecto a la reabsorción del nitrógeno y su disponibilidad. En algunos casos, la reabsorción no cambia con la disponibilidad de nitrógeno en el suelo (Birk \& Vitousek 1986, Schlesinger et al. 1989), en otros aumenta con los aumentos en la disponibilidad (Chapin \& Kedrowski 1983, Nambiar \& Fife 1991) y en otros aumenta con la disminución de la disponibilidad (Small 1972, Boerner 1984, Pugnaire \& Chapin 1993).

A pesar de que la reabsorción de $\mathrm{P}$ foliar no fue significativamente distinta entre las especies del BMA y las del BMB, en este último bosque el porcentaje de reabsorción de $\mathrm{P}$ foliar tendió a ser más alto mientras que las concentraciones de $\mathrm{P}$ disponible en el suelo (Bray II) fueron más bajas. Una mayor reabsorción de $\mathrm{P}$ foliar bajo condiciones de bajo suministro de $\mathrm{P}$ en el suelo, podría tener un valor adaptativo en particular en suelos derivados de cenizas volcánicas donde el $\mathrm{P}$, una vez incorporado a los suelos a través de la hojarasca, tiende a ser ab- sorbido por las alofanas (Vitousek \& Sanford 1986). Cuando la reabsorción de $P$ foliar se expresó como función de la concentración en hojas maduras de diferentes tipos de bosques (Fig. 1), se encontró una correlación negativa y estadísticamente significativa (Spearman $\mathrm{r}=$ -0.5569; p<0.10). Al parecer, en especies de bosques montanos, los porcentajes de reabsorción de $\mathrm{P}$ foliar, estarían controlados parcialmente por la disponibilidad del elemento como ha sido también sugerido para otros bosques tropicales (Vitousek \& Sanford 1986). Este resultado contrasta con lo observado en árboles siempreverdes y caducifolios de la taiga (Chapin \& Kedrowski 1983), del Himalaya (Rahlan \& Sigh 1987) o de la Península Ibérica (Del Arco et al. 1991), donde la reabsorción de P no varió en forma consistente con la disponibilidad.

Escleromorfismo y tasas de reabsorción: cuando se consideraron las diez especies en conjunto, no se observaron relaciones estadísticamente significativas entre la concentración de $\mathrm{N}$ o $\mathrm{P}$ foliar y el área foliar específica $(\mathrm{cm} 2 / \mathrm{g})$. Sin embargo, cuando esta relación se presenta para un número mayor de especies de

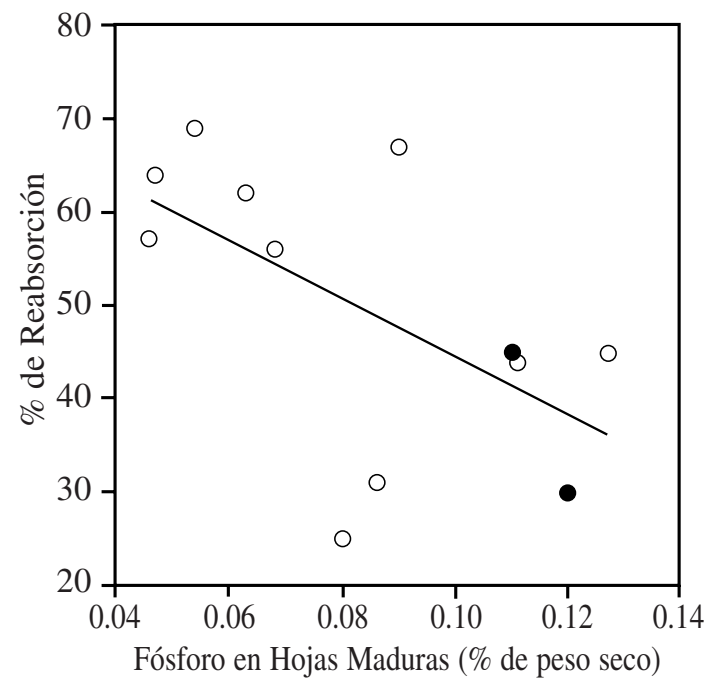

Fig. 1. Relación entre la concentración de fósforo en hojas maduras de especies de bosques montanos y su porcentaje de reabsorción (Spearman $\mathrm{r}=-0.5569 ; \mathrm{p}<0.10) . \mathrm{O}=\mathrm{Da}-$ tos de Cavelier 1996; $\bullet$ = Datos del presente estudio. 


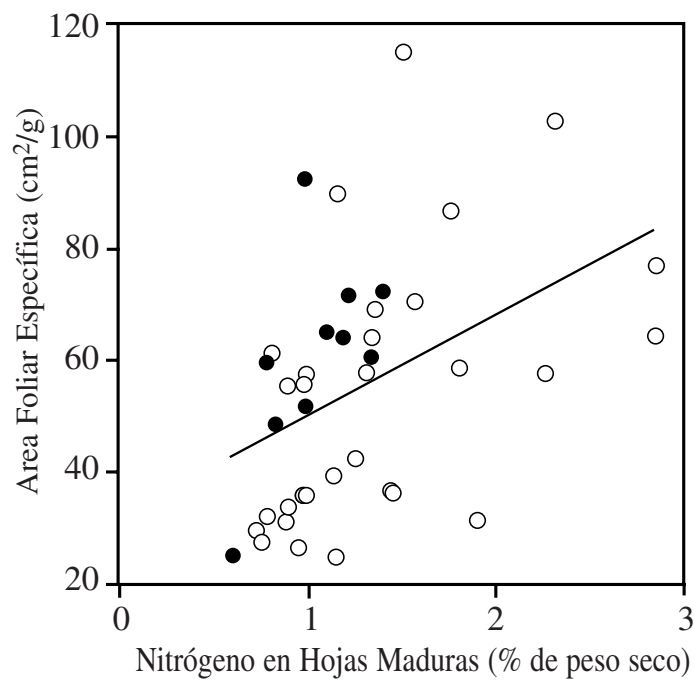

Fig. 2. Relación entre la concentración de nitrógeno en hojas maduras y su área foliar específica (Spearman $\mathrm{r}=$ 0.5498; p<0.001). $O=$ Datos de Cavelier 1986; $\bullet=$ Datos del presente estudio.

árboles de bosques montanos tropicales $(n=40$; Figs. 2 y 3), esta relación pasó a ser estadísticamente significativa (Spearman $\mathrm{r}=0.54$; $\mathrm{p}<0.001$ para $\mathrm{N}$ y $\mathrm{r}=0.33 ; \mathrm{p}<0.05$ para $\mathrm{P})$. Si el área foliar específica es usada como una medida de escleromorfismo (Sobrado \& Medina 1980, Medina 1984), las hojas más escleromórficas de los bosques montanos estarían asociadas a bajas concentraciones de nutrientes foliares por unidad de peso como ha sido reportado para bosques secos tropicales (Montes \& Medina 1977, Kapos 1982) y de "Bana" amazónica (Sobrado \& Medina 1980).

En las diez especies estudiadas, los porcentajes de reabsorción de $\mathrm{N}$ o $\mathrm{P}$ foliar y el área foliar específica, no están relacionados estadísticamente. Sin embargo, esta relación debería ser explorada con un mayor número de especies, pues la falta de correlación puede ser un efecto del tamaño de la muestra como parece ser el caso entre concentración de nutrientes y área foliar específica. En este sentido es interesante recordar que cuando se consideraron los porcentajes de reabsorción de $\mathrm{P}$ en 12 bosques montanos tropicales, se encontró que los bosques con una menor con-

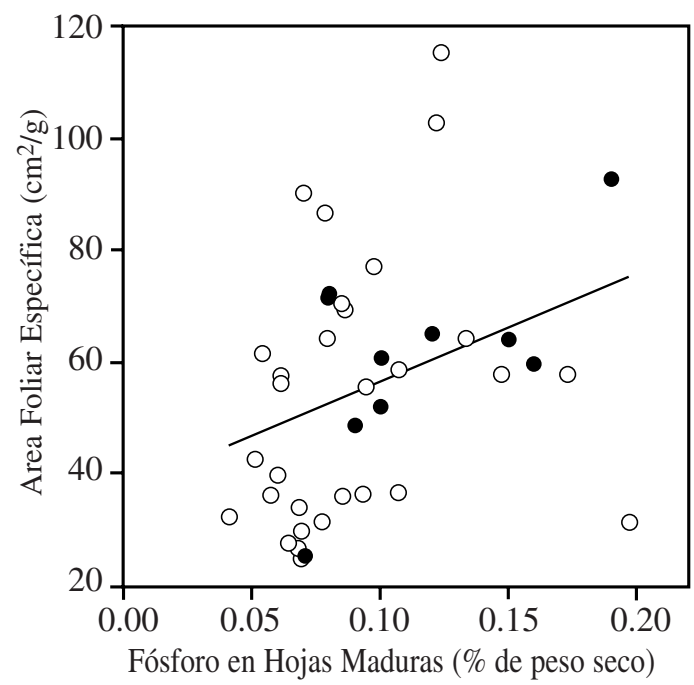

Fig. 3. Relación entre la concentración de fósforo en hojas maduras y su área foliar específica (Spearman $\mathrm{r}=0.5498$; $\mathrm{p}<0.001)$. $\bigcirc=$ Datos de Cavelier 1986; $\bullet=$ Datos del presente estudio.

centración de nutrientes foliares tenían un mayor porcentaje de reabsorción, relación que no fue encontrada para las diez especies de árboles de este estudio.

En resumen, los porcentajes de reabsorción de $\mathrm{N}$ foliar no presentaron variaciones significativas entre tipos de bosques y especies y no parecieran responder a la disponibilidad de los elementos en el suelo u hojas maduras. En contraste, los porcentajes de reabsorción de $\mathrm{P}$ foliar, parecen responder a las variaciones en la disponibilidad del elemento como ha sido reportado en otros estudios.

\section{AGRADECIMIENTOS}

Este trabajo se llevó a cabo gracias a la financiación y apoyo logístico de la Corporación Autonoma Regional de Risaralda (CARDER), a la colaboración de Eduardo Londoño y Jorge Marulanda directores del Parque Regional Natural Ucumarí y de la Estación La Suiza respectivamente. Queremos dejar constancia de nuestro agradecimiento a Martha Galeano del Instituto de Ciencias Naturales y a Gilberto 
Maecha por la determinación del material botánico, a Hernado Acosta, Jaime A. Cabrera y Sebastián Londoño por su colaboración en el trabajo de campo.

\section{RESUMEN}

Se determinaron las concentraciones y los porcentajes de reabsorción de nitrógeno y fósforo foliar en cinco especies de árboles de un bosque montano bajo (BMB) a 1850 m.s.n.m. y en cinco de un bosque montano alto (BMA) a 2800 m.s.n.m en la Cordillera Central colombiana, asi como la disponibilidad de nitrógeno y fósforo del suelo. Los porcentajes de reabsorción de $\mathrm{N}$ y $\mathrm{P}$ no fueron significativamente diferentes entre las especies de estos dos bosques. A pesar de esto, se observó que la reabsorción de $\mathrm{P}$ foliar tendió a ser mayor en las especies del BMB donde la concentración de $\mathrm{P}$ en el suelo fue significativamente más baja (3.2 ppm vs. 9.0 ppm). Cuando la reabsorción de $\mathrm{P}$ foliar se expresó como función de la concentración en hojas maduras en 12 tipos de bosques montanos tropicales, se encontró una correlación negativa y estadísticamente significativa. Estos resultados sugieren que los porcentajes de reabsorción de $\mathrm{P}$ foliar en bosques montanos estarían controlados parcialmente por la disponibilidad del elemento.

\section{REFERENCIAS}

Alexander, E. B. \& J. Pichott. 1979. Soil organic matter in relation to altitude in equatorial Colombia. Turrialba 29: 183-188.

Allen, S. E., H.M. Grimshaw, J.A. Parkinson \& C. Quarmby. 1974. Chemical Analysis of Ecological Materials. Wiley, Nueva York. 565 p.

Birk, E. J. \& P.M. Vitousek. 1986. Nitrogen availability and nitrogen use efficiency in loblolly pine stands. Ecology 67: 69-79.

Boerner, R. E. J. 1984. Foliar nutrient dynamics and nutrient use efficiency of four deciduos tree species in relation to site fertility. J. App. Ecol. 21: 1029-1040.

Cavelier, J. 1996. Environmental factors and ecophysiological processes along altitudinal gradients in wet tropical mountains, p. 399-439. In S.S. Mulkey, R.L. Chazdon, \& A.P. Smith. (ed.). Tropical forest plant ecophysiology. Chapman \& Hall, Nueva York.

Cavelier, J. 1986. Relaciones hídricas y de nutrientes en bosques enanos nublados tropicales. Tesis de Maestría, Universidad de los Andes, Merida, Venezuela.
Cleef, A. M. \& O. Rangel. 1984. La vegetación de las selvas del transecto Buritaca, p. 267-406. In T. van der Hammen \& P.M. Ruiz. (ed.). La Sierra Nevada de Santa Marta (Colombia). J. Cramer, Berlín.

Chapin, F. S. 1980. The mineral nutrition of wild plants. Ann. Rev. Ecol. Syst. 11: 233-260.

Chapin, F. S. \& R.A. Kedrowski. 1983. Seasonal Changes in nitrogen and phosphorus fractions and autumn retranslocation in evergreen and deciduous taiga trees. Ecology 64: 376-391.

Charley, J. L. \& B.N. Richards. 1983. Nutrient allocation in plant communities: mineral cycling in terrestrial ecosystems, p. 5-45. In O.L Lange, P.S. Nobel, C.B. Osmond, \& H. Ziegler. (ed.). Physiological plant ecology. Ecosystem processes: Mineral cycling, productivity and man`s influence. Vol. IV. Springer Verlag, Nueva York.

Del Arco, J. M., A. Escudero \& B. Garrido. 1991. Effects of site characteristics nitrogen retranslocation from senescing leaves. Ecology 721: 701-708.

Edwards, P. J. 1982. Studies of mineral cycling in a montane rain forest of New Guinea. V. Rates of cycling in throughfall and litter fall. J. Ecol. 70: 807-827.

Fassbender, H. W. \& V. Grimm. 1981. Ciclos biogeoquímicos en un ecosistema forestal de los Andes Occidentales de Venezuela. II. Producción y descomposición de los estudios vegetales. Turrialba 31: 39-47.

Grieve, I. C., J. Proctor \& S.A. Cousins. 1990. Soil variation with altitude on Volcan Barva, Costa Rica. Catena 17: 525-534.

Grubb, P. J. 1977. Control forest growth and distribution on wet tropical mountains. Ann. Rev. Ecol. Syst. 8: 83-107.

Grubb, P. J. 1986. Sclerophylls, pachyphylls and pycnophylls: the nature and significance of hard leaf surfaces, p. 137-250. In B. Juniper \& R. Southwood. (ed.). Insects and the plant surface. Edward Arnold, Londres.

Grubb, P. J. \& P.J. Edwards. 1982. Studies on mineral cycling in a montane rain forest in New Guinea. III. The distribution of mineral elements in the above ground material. J. Ecol. 70: 623-648.

Jordan, C. F. 1985. Nutrient Cycling in Tropical Forest Ecosystem. Wiley, Nueva York. 190 p.

Kapos, V. 1982. An ecological investigation of sclerophylly in two tropical forest. Ph. D. Thesis. Washington University, Washington. 
Leigh, E. G. 1975. Structure and climate in tropical rain forest. Ann. Rev. Ecol. Syst. 6: 67-86.

Loveless, A. R. 1962. Further evidence to support a nutritional interpretation of sclerophylly. Ann. Bot. 26: 551-561.

Loveless, A. R. 1961. A nutritional interpretation of sclerophyllous and mesophytic leaves. Ann. Bot. 25: 164-168.

Marrs, R. H., J. Proctor, A. Heaney \& M.D. Mouthford. 1988. Changes in soil nitrogen-mineralization and nitrification along an altitudinal transect in tropical rain forest in Costa Rica. J. Trop. Ecol. 76: 466-482.

Medina, E. 1984. Nutrient balance and physiological processes at the leaf level, p. 139-154. In E. Medina, H.A. Mooney, \& C. Vasquez-Yañes. (ed.). Physiology of plants of the wet tropics. W. Junk, La Haya, Holanda.

Medina, E., E. Cuevas \& P.L. Weaver. 1981. Composición foliar y transpiración de especies leñosas del Pico del Este, Sierra de Luquillo, Puerto Rico. Acta Cient. Venezolana 32: 159-165.

Montes, R. \& E. Medina. 1977. Seasonal changes in nutrient content of leaves of savanna trees with different ecological behavior. Geo. Eco. Trop. 1: 295-307.

Nambiar, E. K. S. \& D. N. Fife. 1991. Nutrient retranslocation in temperate conifers. Tree Physiol. 9: 185207.

Olsen, S. R. \& S.R. Sommers. 1982. Phosphorus, p. 403430. In A.L. Page (ed.). Methods of soil analysis, part 2 , chemical and microbiological properties. American Society of Agronomy, Madison, Wisconsin.

Proctor, J., C. Phillips, G.K. Duff, A. Heaney \& F.M. Robertson. 1989. Ecological studies on Gunung Silam, a small ultrabasic mountain in Sabah, Malaysia. II. Some forest processes. J. Ecol. 77: 317-331.

Pugnaire, F. I. \& F.S. Chapin. 1993. Controls over nutrient resorption from leaves evergreen Mediterranean species. Ecology 74: 124-129.

Rahlan, P. K. \& S.P. Singh. 1987. Dynamics of nutrients of leaf mass in Central Himalayan forest trees and Shrubs. Ecology 68: 1974-1983.

Robertson, G. P. 1989. Nitrification and denitrification in humid tropical ecosystems: potential controls on nitrogen retention, p. 55-69. In J. Proctor. (ed.). Mineral Nutrients in Tropical Forest and Savanna Ecosystems. Blackwell Scientific Publications, Oxford.
Robertson, G. P. 1984. Nitrification and nitrogen mineralization in lowland rainforest succession in Costa Rica, Central America. Oecologia 61: 99-104.

Robertson, G. P. \& P.M. Vitousek. 1981. Nitrification potentials in primary and secondary succession. Ecology 62: 376-386.

Sarmiento, G. 1984. The Ecology of Neotropical Savannas. Harvard University, Cambridge. 235 p.

Schlesinger, W. H., E.H. de Lucia \& W.D. Billings. 1989. Nutrient use efficiency of woody plants on contrasting soils in the western Great Basin, Nevada. Ecology 70: 105-113.

Schubert, G. \& E. Medina. 1982. Evidence of quaternary glaciation in the Dominican Republic: some implications for Caribbean paleontology. Palaeogeog. Palaeoclim. Palaeoecol. 39: 281-294.

Small, E. 1972. Photosynthetic rates in relation to nitrogen cycling as an adaptation to nutrient deficiency in peat bog plant. Can. J. Bot. 50: 2227-2233.

Sobrado, M. A. \& E. Medina. 1980. General morphology, anatomical structure, and nutrient content of sclerophyllous leaves of the Bana Vegetation of Amazonas. Oecologia 45: 341-345.

Steinhardt, U. 1979. Untersuchungen uber den Wasserand Nahrstof- fhaushalt eines andien Wolkenwaldes in Venezuela. Gottinger Bodenkundliche Berichte 56: 185.

Stoker, O. 1931. Transpiration und Wasserhaushalt in verschiedenen klimazonen. I. Untersuchungen and der artischen Baumgrenze in Schwedisch Lappland. Jahrb wiss. Bot. 75: 494-549.

Sugden, A. M. 1985. Leaf anatomy in a Venezuelan montane forest. Bot. J. Linn. Soc. 90: 231-241.

Tanner, E. V. J. 1977a. Four montane rain forest of Jamaica: a quantitative characterization of the floristics, the soils, and the foliar mineral levels, and a discussion of the interrelations. J. Ecol. 65: 883-918.

Tanner, E. V. J. 1977b. Mineral cycling in montane rain forest in Jamaica. Ph. D. Thesis, University of Cambridge, Cambridge.

Tanner, E. V. J. \& V. Kapos. 1982. Leaf structure of Jamaican upper montane rain forest trees. Biotropica 14: 16-24.

Thouret, J. C. 1983. Presentación geológica y geomorfoestructural, p. 48-55. In T. van der Hammen, A. P. 
Preciado \& P. Pinto. (ed.). Studies on tropical Andean ecosystems. La Cordillera Central colombiana. Transecto Parque Los Nevados (introducción y datos iniciales). Vol. 1. J. Cramer, Berlín.

Thouret, J. C. 1989. Suelos de la Cordillera Central, transecto Parque Los Nevados, p. 293-441. In T. van der Hammen, S. Diaz-Piedrahita \& V. J. Alvarez. (ed.). Studies on tropical Andean ecosystems. La Cordillera Central colombiana. Transecto Parque de los $\mathrm{Ne}$ vados. Vol. 3. J. Cramer, Berlín.

van der Hammen, T. 1984. Temperaturas de suelos en el transecto Buritaca La Cumbre, p. 67-74. In T. van der Hammen \& P.M. Ruiz. (ed.). Studies on tropical Andean ecosystems. La Sierra Nevada de Santa Marta (Colombia). Transecto Buritaca La Cumbre.Vol 2. J. Cramer, Berlín.
Veneklaas, E. J. 1991. Litterfall and nutrient fluxes in two montane tropical rainforest, Colombia. J. Trop. Ecol. 7: $319-336$

Vitousek, P. M. 1984. Litterfall, nutrient cycling, and nutrient limitation in tropical forests. Ecology 65: 285-298.

Vitousek, P. M., P.A. Matson \& D.R. Turner. 1988. Elevational and age gradients in Hawaiian montane rainforest: foliar and soil nutrients. Oecologia 77: 565-570.

Vitousek, P. M. \& R.L. Sanford. 1986. Nutrient Cycling in moist tropical forest. Ann. Rev. Ecol. Syst. 17: 137-167.

Weaver, P. L., E. Medina, D. Pool, K. Dugger, J. GonzálezLiboy \& E. Cuevas. 1986. Ecological observations in the dwarf cloud forest of the Luquillo Mountains in Puerto Rico. Biotropica 18: 79-85. 